\title{
Adaptive practices in heart failure care teams: implications for patient-centered care in the context of complexity
}

This article was published in the following Dove Press journal:

Journal of Multidisciplinary Healthcare

19 August 2015

Number of times this article has been viewed

\section{Glendon R Tait' \\ Joanna Bates ${ }^{2}$ \\ Kori A LaDonna ${ }^{3}$ \\ Valerie N Schulz ${ }^{4}$ \\ Patricia H Strachan ${ }^{5}$ \\ Allan McDougall ${ }^{3}$ \\ Lorelei Lingard ${ }^{3}$}

'Department of Psychiatry and Division of Medical Education, Dalhousie University, Halifax, NS, ${ }^{2}$ Centre for Health Education Scholarship, Vancouver General Hospital, Vancouver, BC, ${ }^{3}$ Centre for Education Research and Innovation, Schulich School of Medicine and Dentistry, Western University, ${ }^{4}$ Palliative Care, London Health Sciences Centre, University Hospital, London; ${ }^{5}$ School of Nursing, McMaster University, Hamilton, ON, Canada
Correspondence: Glendon R Tait Department of Psychiatry, Dalhousie University, 8th Floor, Abbey J Lane Memorial Building, 5909 Veterans' Memorial Lane, Halifax, NS B3H 2E2, Canada

Tel +l 9024732492

Email glendon.tait@utoronto.ca
Background: Heart failure (HF), one of the three leading causes of death, is a chronic, progressive, incurable disease. There is growing support for integration of palliative care's holistic approach to suffering, but insufficient understanding of how this would happen in the complex team context of HF care. This study examined how HF care teams, as defined by patients, work together to provide care to patients with advanced disease.

Methods: Team members were identified by each participating patient, generating team sampling units (TSUs) for each patient. Drawn from five study sites in three Canadian provinces, our dataset consists of 209 interviews from 50 TSUs. Drawing on a theoretical framing of HF teams as complex adaptive systems (CAS), interviews were analyzed using the constant comparative method associated with constructivist grounded theory.

Results: This paper centers on the dominant theme of system practices, how HF care delivery is reported to work organizationally, socially, and practically, and describes two subthemes: "the way things work around here", which were commonplace, routine ways of doing things, and "the way we make things work around here", which were more conscious, effortful adaptations to usual practice in response to emergent needs. An adaptive practice, often a small alteration to routine, could have amplified effects beyond those intended by the innovating team member and could extend to other settings.

Conclusion: Adaptive practices emerged unpredictably and were variably experienced by team members. Our study offers an empirically grounded explanation of how HF care teams selforganize and how adaptive practices emerge from nonlinear interdependencies among diverse agents. We use these insights to reframe the question of palliative care integration, to ask how best to foster palliative care-aligned adaptive practices in HF care. This work has implications for health care's growing challenge of providing care to those with chronic medical illness in complex, team-based settings.

Keywords: palliative care, qualitative, complex adaptive system, multimorbidity, health care teams

\section{Introduction}

Heart failure (HF) is a chronic, progressive, incurable disease and one of the three leading causes of death in Canada. There is growing policy support for the integration of palliative care for patients with advanced HF, in order to prevent and relieve suffering by using a holistic approach to address patients' physical, psychosocial, and spiritual needs. ${ }^{1-5}$ Research informing this policy shift primarily arises from survey studies and meta-reviews, which have yielded consistent descriptions of patients', caregivers' and health care providers' individual knowledge, needs, and attitudes regarding palliative care in HF. ${ }^{1-3,6-8}$ However, this information about individual elements in a complex 
system is insufficient, because integration cannot be achieved by individual providers. It will require collaborative effort by the patients, caregivers, community members, and a host of health care professionals who constitute the HF care team. While the term HF care team implies coherence and stability, HF care is delivered by a shifting roster of individuals from different specialties and institutions, who are often not as well coordinated or connected as the term team would imply.

In this regard, HF care represents an iconic instance of one of contemporary health care's most intractable problems: how to coordinate care for the complex medically ill. ${ }^{9-12}$ Unless they take into account the complex, dynamic care context, efforts to improve care for this growing group of patients are likely to fail. Therefore, while a key impetus for our study is to inform appropriate palliative care integration efforts in $\mathrm{HF}$ care, we also aim to add to the general understanding of how care is delivered to the complex medically ill. ${ }^{13,14}$

The HF team includes patients, caregivers, and health care providers from different specialties, with their different clinical goals and priorities, different resources, and different health care and organizational systems. The purpose of this study was to understand how inherently complex HF teams work, specifically the dynamic practices and negotiations among HF team members caring for patients with advanced HF. This understanding is required to inform efforts to integrate palliative care and HF care in robust and sustainable ways and to address the broader challenge of providing care in chronic, advanced, comorbid illness.

\section{Theoretical framework}

Complex adaptive system (CAS) theory orients our work to appreciate the multifactorial, negotiated nature of teamwork in advanced HF care. A CAS is an open system composed of multiple agents; these agents can include people, policies, equipments, technologies, institutions, and their values and practices. A CAS is greater than the sum of its parts, and a robust understanding cannot be had by reducing it to its individual components or agents, which are entangled and interact dynamically. ${ }^{15,16}$ For example, in the HF care team, relationships are not simply reducible to division of labor or specialization. Interactions within and between roles exist, are shaped by professional regulations, organizational routines, and interpersonal relations and, therefore, give rise to unforeseeable events. Furthermore, as an open system, an HF care CAS is constantly interacting and exchanging information with the external environment that includes other systems and subsystems such as the palliative care CAS. ${ }^{17,18}$

CAS are nonlinear, meaning that small disturbances or changes in the system can produce large and unpredictable consequences. This raises the question of whether predictable change is achievable through imposition of external mandates, such as new protocols for integrating palliative care. Rather, change in a CAS often occurs unpredictably, through the process of self-organization, in which local interactions between agents affect the broader system, often in unintended ways. ${ }^{19}$ This dynamic process has been described as "the responsive negotiating among individuals in particular, contingent situations". ${ }^{20}$ The changes that appear as new properties and patterns in a CAS are the result of the emergent behavior of that system.

In contrast to a CAS approach, change efforts in health care have commonly treated the health care system as an amalgam of parts that relate in predictable ways. Such an approach is drawn from and suitable for manufacturing and mechanical problems, such as building or fixing a car, which are characterized as complicated or linear. But a mechanistic approach is rarely appropriate for the complexity of health care delivery, in which parts are not fully knowable, interact unpredictably, and can produce unintended outcomes. ${ }^{21} \mathrm{HF}$ care is such a complex problem: it is enacted by a diverse and only partly explicit set of individuals who interact in different ways across highly varied contexts of care provision, around a condition that is characterized by an undulating nature and unpredictable prognosis, in support of patients with variable understanding of and compliance with treatment regimens. CAS theory has been applied productively to the question of why practice interventions succeed or fail in the setting of primary care teams, but it has not yet been used as a lens to examine the coordination of care for a patient with advanced HF. ${ }^{13,14}$

\section{Methods}

This paper reports results from a multicenter, qualitative study exploring the practices of HF care teams at five study sites in three Canadian provinces: Ontario, Nova Scotia, and British Columbia. The study was approved by the Research Ethics Board at each site.

A more detailed description of our research methodology is presented elsewhere. ${ }^{11}$ We used constructivist grounded theory (CGT) to develop a contextualized description of advanced HF care as it is enacted by teams..$^{22,23}$ The purpose of CGT is to build an explanatory theory grounded in recurring patterns in the dataset, taking into account the constructed nature of reality. CGT assumes that researchers and research participants co-create the theory that arises from research inquiry; therefore, investigator triangulation is critical. We assembled a research team constituted of perspectives and experiences relevant to the issue of integrating palliative care 
into HF care. Our team included social scientists, physicians, and nurses with palliative care, HF care, psychiatry, and primary care expertise and graduate students from sociology, health policy, and education.

Patients with advanced HF defined as New York Heart Association (NYHA) III or IV were recruited primarily from heart function clinics through a combination of convenience and purposive sampling to participate in semi-structured interviews. We used an innovative, patient-centered methodological approach to build the sampling and recruitment strategy for team members. ${ }^{11}$ Index patients were asked to identify their team members - loosely defined as the individuals who provide them with recurring, supportive, HFrelated care. If index patients consented, their identified care team members were invited to participate in semi-structured interviews. Interviewees became part of team sampling units (TSUs) (Figure 1) - defined as a patient-identified health care team consisting of the patient plus at least two other members. ${ }^{11}$ To ensure confidentiality, each participant was given a pseudonym. Index patients for whom we were unable to recruit at least two team members for interview were not included in the TSU analysis.

Data collection and analysis occurred simultaneously and iteratively. All researchers read a selection of transcripts to create a list of preliminary codes representing recurring themes or patterns in the data. As the analysis matured, smaller coding groups focused on particular codes, applying them to representative TSU transcripts to explore their fit and to more closely define the recurring processes they included. Our iterative process allowed new questions to be added to the interview guide to probe subsequent participants about emerging patterns in the analysis and afforded the ability to use theoretical sampling to purposefully recruit participants to explore emerging analytical ideas. ${ }^{22,23}$ For example, we sampled more socially frail NYHA class III and IV patients in later study sites, in order to further explore the emerging role of community members on HF care teams and their implications for how care evolves as patient status worsens. We also purposively recruited more complex HF patients as the study progressed, due to our analytical speculation that our early recruitment had captured more stable NYHA class III and IV patients and that complexity was a key factor in patients' and their care teams' expectations and experiences of care. Sufficiency of data was determined when ongoing analysis did not yield new insights about major themes. ${ }^{24}$

For the analysis reported in this paper, a small coding group (GRT, JB, KAL, LL) analyzed the data using a constant comparative method to refine and apply the codes and categories, returning to particular instances to discuss discrepancies and refine understanding of relationships among categories. ${ }^{22,23}$ Attention was paid not only to patterns within individual interviews but also to patterns across TSUs, particularly where divergent perspectives or experiences were apparent. The final, elaborated set of categories arising from

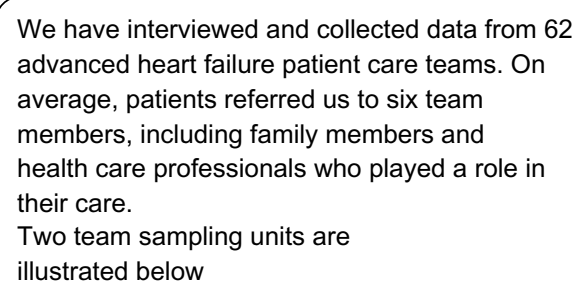

Team 1: Kevin

A 65-75 year old man whose wife, heart specialist, and heart failure clinic nurse practitioner were interviewed. He also identified his, primary care physician, who was unavailable for an interview.

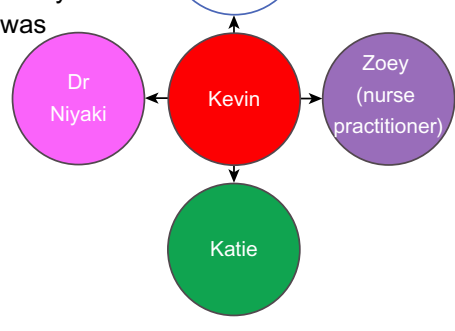

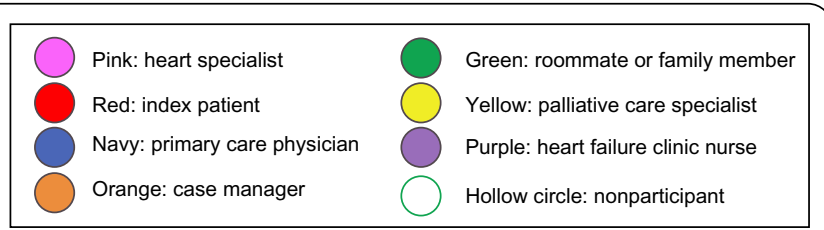

Team 2: Irene

A 50-55 year old woman whose roommate, primary care physician and heart specialist were also interviewed. She also identified her case manager, who was unavailable for an interview.

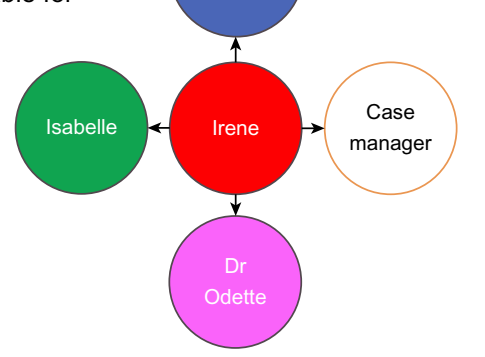

Figure I Team sampling unit. 
this group's analytical process was applied by one researcher KAL to subsequent sets of data collected later in the study timeline, until all data were coded. NVivo, a qualitative research software program, was used to organize and manage the data.

\section{Results}

Our dataset consists of 209 interviews from 50 TSUs, including a variety of health professionals, family members, and other caregivers (Table 1). Data excerpts in this paper are referenced according to the study site, participant pseudonym, participant role, and index patient name, eg, Site 3: Diane, caregiver; Donna, patient.

While TSU participants reported many details regarding the biomedical aspects of HF care, their interview narratives focused most intently on the organizational, social, and practical aspects of how the HF care system works. In our analysis, we captured this dominant feature in the thematic category of system practices. We identified two distinct subthemes: "the way things work around here", which were commonplace, routine ways of doing things, and "the way we make things work around here", which were more conscious, effortful practices that adapted the

Table I Care team members, according to role.*

\begin{tabular}{ll}
\hline Total number of unique individual team members & 152 \\
Caregiver/supportive person & 55 \\
Spouse & 21 \\
Family members & 22 \\
Friend & 8 \\
Taxi driver & 1 \\
Spiritual support & 1 \\
House keeper & 1 \\
Housing/residential director & 1 \\
Family physician & 25 \\
Nurses & 18 \\
Specialist physician & 35 \\
Cardiologist & 24 \\
Nephrologist & 23 \\
Surgeon & 4 \\
Palliative care & 2 \\
Urologist & 1 \\
Geriatrician & 1 \\
Allied health/other health professionals & 15 \\
Pharmacist & 5 \\
Physiotherapist & 1 \\
Care aid & 4 \\
Counselor & 1 \\
Dentist & 1 \\
Dietician & 1 \\
Clinic technician & 1 \\
Social worker & 1 \\
\hline
\end{tabular}

Note: *Some care team members were identified by and formed part of a team sampling unit (TSU) of more than one patient. usual routine when it was perceived to be insufficient or ineffective. In coding, we based our analytical decisions as to whether care was usual or had been adapted according the perspective of participants, as articulated in the data. In what follows, we illustrate briefly the theme "the way things work around here" to provide context for more in-depth consideration of the novel contribution of our research: how and why the usual routine may be adapted by HF care teams, and what such adaptive practices mean for team-based HF care generally and the integration of palliative and acute HF care specifically.

\section{The way things work around here}

This category refers to features of the current HF care delivery system that participants described as commonplace routines and reported matter-of-factly as "just the way things are done". These were not descriptions of how things should work but, rather, of the usual practice. The interview transcripts abounded with references to commonplace features of the HF care system. Such features included, but were not limited to, test ordering practices, documentation systems, referral and consultation structures, procedures for patient assessment, and clinical scheduling routines. However, "around here" was highly contextualized to each clinical environment and was influenced by factors, including provincial health care structures and funding variations and how clinicians set up their practices and approached HF care. This heterogeneity of context offers a rich variety of instances of usual organizational practices of providing HF care.

Appointment scheduling was a recurrent example of a commonplace, usual routine, which we will use to illustrate this subcategory of system practices. Many participants described their experience with clinic and private practice scheduling systems, systems that were all based on organizing multiple, time-limited interactions between care professionals and patients. Health care appointments were invariably described as having a specific duration and pace, which framed both the overall structure of a clinic's day and, to some degree, the purpose and process the individual encounters. One patient described a visit to the HF clinic he attended:

You walk down to where the reception is you say, yeah, I'm here. Your health card and boom, yeah, just a minute, they do something in the computer and then they go down and say follow me, the room is empty and they put you in a room. The next thing you know, people are popping in and I thought, this is good ... It's very well done, yeah, and it's fast. (Site 3: Norman, Patient) 
Another patient's caregiver discussed the issue of clinic scheduling from a very different perspective:

To make an appointment, there's no way you can get a same

day appointment. There's no way, most of the time, that

you can even get an appointment in the same week (Site 2:

Isabelle, Caregiver of Irene).

These two examples illustrate the variation within descriptions of the commonplace system practice of clinic scheduling. Clinic scheduling was recognized as having both positive effects, such as efficiency and increased access, and negative effects, not only for patients but also for health professionals. Commonplace clinic structures with their timelimited, focused interactions were perceived as particularly problematic for HF patients whose multiple needs were not perceived as amenable to the one-problem-per-visit model of primary care standard in many of our study settings:

In this fee-for-service environment, it doesn't encourage sitting back and relaxing, conversation about, well, how's your life going along, and things like that. It's more of one problem per visit kind of thing. Now, many, many patients come in with more than one problem ... I've given up trying to explain to people that, when they have more than one problem, that interferes with properly addressing each problem. I've given up doing that, because people just simply don't understand. (Site 1: Dr. Rutgers, Family Physician of Reema)

The routine of appointment scheduling varied with contextual features such as the standard duration of an appointment, the flexibility possible in the daily appointment system, and the ability of patients to initiate appointments (in contrast to provider-initiated appointments). However, only when such variations were expressed, according to the participant's experience, as conscious and effortful diversions from the usual routine, did we code them in the next subcategory.

\section{How we make things work around here}

Our data included descriptions of individuals and teams working more effortfully to make things work when the usual practice was perceived to be insufficient or inappropriate. We defined adaptive system practices as any instances in which team members described altering the usual way of doing things in order to be responsive to emergent needs, usually patient needs.

To illustrate, consider again the instance of appointment scheduling. Many participants explicitly contrasted the usual with an adaptive practice in their narratives. As one caregiver explained:

He [the patient] makes an appointment usually, but if he wasn't feeling well when he got up in the morning, if we felt he should be in to see someone, if I call they'll [FP clinic] say come right in. It's come right in, or he's got a few in the waiting room so come in an hour or something (Site 1: Annie, Caregiver of Albert).

In this example, the usual requirement to have an appointment is adapted in the face of Albert's emergent situation. The adaptation is based on his family physician's perception and trust that Albert is able to accurately assess his own health state. Our data included examples from several teams in which HF patients were empowered to direct their own care according to their perceived need for medical assistance. As one HF nurse practitioner explained:

Sylvan is pretty good, and that was the same with Farida, very good at self-managing their heart failure. So, they would adjust their own diuretic needs, based on their symptoms or their weight. They were very good at managing their own symptoms. They would call the clinic and you would know, when they called the clinic, they were sick.

(Site 1: Barbara, HF Clinic Nurse Practitioner of Sylvan and Farida.)

Patients and health professionals described other instances of altering the usual clinic scheduling practices to respond to patient need. For instance, one physician provided his home phone number to a patient: "If I can't get him at the office, I can get him at home" (Site 5: Finlay, Patient), and the usual structure for a follow-up clinic visit could be adapted to offer a patient more attention and time as required:

We scheduled her that she was ... the last person of the day, [so] that we would have the time to spend with her. Our appointments with her, even though it was a followup, would end up being an hour because we were dealing with more conversation, coping strategies, just being there for her. (Site 3: Odessa, HF Clinic Nurse Practitioner of Ophelia)

In some situations, participants described making things work in a particular situation by discarding the usual clinic structure altogether in favor of home visits. For instance, some providers reported making "house calls to keep ... people in their homes longer" (Site 1: Dr. Akamura, Family Physician of Albert) when patients had reached a stage of extreme illness or frailty. 
Like usual system practices, adaptive practices were variably experienced and understood, with participants' evaluation of their value depending on context and point of view. To illustrate, consider the example of the system practice of referral and consultation. Participants reported a usual way or set of expectations of how referral and consultation would be conducted in the HF care system as well as a number of adaptive practices that altered the usual to make referral and consultation work in particular situations. How such adaptive practices were experienced by participants depended on their perspective and role in the referral and consultation system. As one family physician explained:

Theoretically, the specialists are supposed to be consultants.

In other words, they answer questions to the family doctor and make recommendations, and then the family doctor continues to manage the patient with this knowledge given to them by the specialist. However, there are some specialties where the specialist basically takes over the care of the patient, and the patient disappears. (Site 1: Dr. Rutgers, Family Physician of Reema)

A cardiologist described the rationale, from his point of view, for the adaptive practice of a specialist taking over care of the referred patient:

It depends on the severity of the illness and whether or not I think they can be managed appropriately by their family doctor. And so the factors I consider are: where are they from, what are the patient's individual supports, what do I know about the family doctor? Is it somebody I know? Is it a comprehensive, caring [doctor who] will spend the necessary time? Or, is this the family doctor where my impression is that they probably ... are not spending the appropriate time [to] follow up the patient as closely as needs be? (Site 1: Dr. Spelling, Cardiologist of Sylvan)

This explanation represents the adaptive practice of taking over a referred patient as a necessary response when the family physician is perceived as unlikely to follow up with the HF care appropriately, either due to time or expertise. For HF patients, who in our study often had more than three health professional care providers, adaptive system practices regarding referral and consultation were particularly salient. Whether and how team members decide to adapt the usual referring and consulting practice varied widely in our data, but was usually portrayed to be based on an assessment of patient need.

Instances of adaptive referral and consultation practice in our data provide insight into how team members seek to ensure effective patient care in a complex, shared care context. They also reveal sites of tension between medical specialties, such as nephrology and cardiology, whose treatment priorities may vary. As one nephrologist admitted, the interface between these two specialties is a site of delicate cooperation and tacit adaptive practice in HF care:

We both agree, both programs agree, about salt restriction, but you'll find that the nephrologist don't by and large agree with water restriction. We think that that makes a bit of a difference to maintaining renal function. That would just be a typical communication or direct patient management issue that we might differ on and we have to be very careful as to how we handle slight differences of opinion like that in dealing with the patients .... I think we acknowledge that the cardiologists usually in these circumstances are the prime caregivers. We're talking about a heart failure patient, we're not talking about a renal failure patient with fluid overload, so they're probably obliged to follow the cardiologist directions, although I might tend to whisper in their ear and tell them to lie to the cardiologist about how much liquid they drink. (Site 4: Dr. Vextor, Nephrologist of Victor)

While small, such innovations could have amplified effects beyond their immediate setting and beyond the innovator's intended consequences. For instance, the practice of retaining referred HF patients could lead patients to feel that their HF clinic was their primary care provider:

I haven't seen my family doctor in a pretty long time .... I don't need him as much because they'd (HF clinic and private labs) look after my functions ... everybody else looks after me. (Site 5: Carmen, Patient).

This was reported to have two effects. First, family physicians could lose touch with the patient:

So, theoretically, I'm supposed to be the coordinator of her care ... the Heart Function Clinic should only be interested, and focus, on her heart function. And, if there are any other issues that are identified by her, then they should send her back to me, for me to address it .... But, sometimes, clinics and their specialists will take up something and run with it. A lot of the times, they'll include me in the information circle, but sometimes they don't. And, patients will come in and say, oh, I got this done, or I got that done, or whatever. I find out that way. (Site 1: Dr. Rutgers, Family Physician of Reema)

Second, the HF clinic could find itself addressing patients' primary care issues, with implications for their capacity 
to fulfill their function of HF care. As one HF clinic nurse explained:

What ends up happening a lot is they call you because they have a sore leg or they fell ... because we're accessible. But we'll say with that, really, you should see your family doctor or speak to your family doctor about that ... (Site 1: Felicia, HF Clinic Nurse of Oscar and Tatsumi.)

\section{Unpredictability of adaptive practices}

Our participants' reports of how they make things work around here were almost always presented as purposeful problem solving: when faced with a disturbance or emergent need, some team members found new ways of making the system work. Such adaptive practices, while recurrent in our dataset, were not predictable. Consider the situation of patient immobility, experienced by both Ida and Irene. Ida's family physician described an adaptive practice - home visits - in response to Ida's emerging inability to fit into the usual practice of scheduling patients to come to clinic for their appointments:

... She's quite limited in her mobility ... I visited her during the winter because I really didn't want her out during the winter, but from last year to this year, she has slowed down quite a bit ... I just actually called to see how she was doing with regard to another problem, and said, if she was still having trouble, to book me in for a home visit because I think she's getting to that point that she will need home visits. (Site 1: Dr. Isadora, Family Physician of Ida.)

In contrast, Irene's family physician recognized that immobility was limiting Irene's ability to fit the usual practice of clinic visits, but did not describe adapting the usual practice in this case:

Getting simple things done is difficult because she can't afford anything and she's not ambulatory .... So a lot of our consistency, just in terms of monitoring even blood work, monitoring weight, it takes a lot to get her in here as you can imagine, because she's not ambulatory .... The reality is that monitoring this type of person without somebody being able to go into the home is extremely difficult. (Site 2:

Dr. Queen, Family Physician of Irene)

As these two examples indicate, similar situations did not predictably yield similarly adaptive responses from team members in our dataset. What produced this difference in response? Was there something about the local context of Ida's HF care team in Site 1 that made home visits more feasible? Did Irene's physician, practicing in Site 2, have other adaptive practices at her disposal to respond to the problem of patient immobility?

\section{Conditions influencing adaptive practices}

In trying to understand the influences that produced variability in adaptive practices, we reanalyzed the adaptive practices category with attention to conditions that appeared to influence these practices. We identified three conditions that interacted to foster adaptation: patient complexity or being unusual, the provider's perspective on complexity, and the relationship or human connection between the patient and the provider.

Participants recurrently described adaptive practices in response to patients who were particularly complex or unusual. While complexity, in the form of having multiple illnesses, was common, particular comorbidities seemed more likely to prompt adaptive practices. Examples included cognitive impairment, mood disorders, or mobility issues, all impacting the patient's ability to participate in or access care in the usual ways.

The definition of and response to patient complexity was in part a function of the health care provider's perspective on and comfort with that complexity. For example, Irene's community cardiologist responded to her complexity by referring her to another provider, while Wendy's HF clinic cardiologist explained that he was well suited to care for her complex array of problems:

She's a very complex patient; she has an unusual form of heart failure, that I think is not necessarily well understood, so she gets into problems when she encounters new care providers who don't really understand her. I have a feeling that's why she really wants to continue to come and see me and appreciates seeing me, because I have been looking after her for quite a significant length of time, I know very well what her problems are, and have quite a bit of experience in how she reacts to adjustments to medications and so on.

(Site 4: Dr. Waide, Cardiologist of Wendy)

Whether a patient was perceived as unusual - characterized by having a disease course outside the anticipated norm - was another factor influencing adaptation. For example, Ophelia was in her 40s and suffered from HF and other complex comorbidities uncommon in someone in her age. The HF clinic nurses reported that they responded to Ophelia's unique emotional needs by scheduling her at the end of the day, allowing for much longer follow-up appointments than was the usual practice. Ophelia was cognizant 
of the extra care and attention from the nurses at the HF clinic:

They all come and sit with me. And, I don't know if it's because they're bored, or it's because I'm just such a character ... quite honestly, you don't have young people in the cardiac [clinic]. So, I guess in some ways I'm just a breath of fresh air, because everyone else is really old. (Site 3: Ophelia, Patient)

As with complexity, being unique was not an objective state, but rather interacted with health care providers' perspective, comfort level, and available resources to sometimes foster adaptation. For example, Carina (Site 4) was a young patient presenting with HF secondary to IV drug use and HIV/AIDS. Her care aide described the ability to access HIV/ AIDs resources by enrolling her in a chronic care home for patients with end-stage HIV disease, resulting in "a higher quality of service" for her than the usual HF care resources could have offered her (Site 4: Cody, Care Aide of Carina). Carina was both complex and unusual, features that were perceived by her care providers as opportunities to access additional care resources to meet her increased needs. In stark contrast, Irene (Site 2), while complex, was seen by all participants in her TSU as not receiving the care she needed. While hypothetical solutions for Irene were mentioned, such as more extensive monitoring by homecare personnel, none were described as being implemented. As her cardiologist put it, Irene's complexity and her incompatibility with the way things usually work caused her to "[fall] through the cracks". Contrasting Carina's and Irene's situations outlines the nonlinear, unpredictable, emergent nature of adaptive practice. The response to complexity in one case was adaptive practices, while apathy and paralysis emerged in the other.

Finally, adaptive practice was influenced by a connection between patient and provider as human beings, beyond their roles as patient and health care provider. As one family doctor acknowledged:

...I suspect it's more internalized than conscious. With Sylvan, I think there was quite a connection. This is wrong, but it's a truth that every doc has; it's really easy to give good care to someone you really like. You can't help but respect Sylvan, and can't help but like him. I'd like to think I gave the same care to everyone, but that's probably not true.

(Site 1: Dr. Smoke, Family Physician of Sylvan)

Similarly, Albert's family physician spoke of a connection that transcended the professional relationship, allowing him to adapt his strategies to fit the patient's persistent noncompliance with his HF management regimens. Interviewed following Albert's death, Dr Akamura was visibly emotional as he acknowledged that

We were clearly friends also. It just transcended it ... He was a wonderful man and that nature was what let him go so far and get away with it, because you would never get angry at him. (Site 1: Dr. Akamura, Family Physician of Albert).

The implication that noncompliance would normally provoke a negative emotional response in the physician was evidenced in many physician interviews; here, the friendship between Albert and Dr Akamura allowed for a different response to Albert as a wonderful man rather than a noncompliant HF patient.

Another intriguing example of the role of human connection in adaptive practice was evident in the interview with Carina's cardiologist, Dr Cameron. He reported feeling frustration and futility caring for IV drug users with HF, acknowledging he may be too hard on them in regard to their noncompliance and lack of knowledge about their condition. However, his usual approach was adapted with Carina:

I was sort of willing to give her some slack .... I kind of had a soft spot in my heart for her, because she reminded me a lot of my sister who was very sick ... until she died. His human connection with Carina caused the cardiologist to alter his usual practice with HF patients who are IV drug users.

The orientation to the patient as a person beyond their HF appeared to be a central condition for adaptation. However, this condition emerged in the unique interaction between the provider and patient: no patients in our dataset reliably provoked this orientation in all their providers. The human connection between Albert and his family physician was not reproduced in Albert's other relationships with health care providers. In fact, he reported other health care relationships where human connection was conspicuously lacking, as in his narrative of a nurse in hospital who refused to respond to his plea to remove a catheter:

I had pain, I was getting pain and she said "you will get used to it." I said look, I can't stand it. I said look, and finally I just called her in and I said, either you take it out or I'm ripping it out. She said, if you rip it out, I'll be putting it back in. She said, "get used to it."

While patient complexity or being unusual, provider perspective and human connection all served to foster adaptive practices in some instances; the interaction of these 
three conditions, as opposed to any one alone, seemed to influence the emergence of adaptive practices.

\section{Discussion}

Adaptive practices are a form of emergence, evidence of what Stacey has called "the responsive negotiating among individuals in particular, contingent situations". ${ }^{20}$ While there are usual ways of organizing and enacting care practices on HF teams, adaptations emerge as individual team members interact and respond to emergent needs, usually of the patient. One of the striking features of our dataset of 50 TSUs is its variety: no two HF patients have the same care experiences, even when they come from the same study site and share apparently similar characteristics such as immobility or cognitive impairment. This variability is a reflection of the underlying process of self-organization, a key component of CAS.

Many team members described tinkering with the usual as a matter of course to keep the system operating. While downstream effects were sometimes described as frustrating to those experiencing them, such as the family physician whose patient "disappears" into the specialist's domain, they were nevertheless presented as largely unavoidable. That is, no participant expressed a desire that the system remain static and predictable; each, however, was likely to express a preference for their own adaptations over those of others.

This tone across the interviews suggests that participants in the HF care system recognize that adaptation is inevitable and neither inherently good nor bad. In CAS terms, we would say that the variability of the adaptive practices we have described is a function of the interdependence of parts in a complex system and the emergent properties that result from the interactions among them. ${ }^{25}$ Lanham argues that this is why a health care organization cannot be understood by attending to its individual components in isolation. ${ }^{14}$ The human components of HF care system - patients, providers, and family members - are dynamic and entangled; for instance, patient complexity only provokes adaptation through its interaction with a team member's perspective and possibly a human connection. ${ }^{15,16}$

Following Kannampallil et al, therefore, we would contend that an authentic understanding of how HF care works is only possible through granular attention to the nature and constraints of such interrelations ${ }^{26}$ Our description of the conditions that influence adaptive practices is an example of attending to interrelations among components in order to understand what is emergent from a system. Our results illustrate starkly that emergence of adaptive practices is nonlinear and unpredictable and their perceived impact is variable. Our findings reflect the CAS premise that, while the components of the system are important - specialty roles, patient characteristics, and clinic policies - their connections and interrelationships are even more important. ${ }^{27}$ Because these connections are nonlinear, they give rise to apparently illogical events, such as a nephrologist advising a HF patient to consume more fluid than recommended by their HF self-care regimen.

The presence of adaptive practices on the HF care team underscores the difficulty of seeking change through topdown mandates in this care setting, such as regulations or guidelines. Such approaches to the integration of palliative care into HF care assume that HF care is a stable target for intervention; our results illustrate that this is not the case. Rather, the regularity of adaptive practices in the HF care teams we interviewed suggests that integration efforts will need to embrace this reality, and may be more likely to emerge spontaneously, through self-organization, than through external intervention, such as guidelines.

Kauffman has pointed out the importance of selforganization to health care change efforts, because it draws our attention to ways in which local interactions between agents affect the broader system even when no agent has the intention to create such effects. ${ }^{19}$ For instance, in our data, when cardiologists retain HF patients for follow-up, those patients over time may shift their primary care expectations onto the HF clinic, inadvertently removing the family physician from their primary care role on that patients' team. This is neither a linear nor a predictable pattern. The patient's distance from the family physician is not caused by HF clinic follow-up per se, nor is it a reliable outcome of HF clinic attendance. However, it may, through amplification via feedback loops, influence higher level issues such as HF clinic capacity or primary care utilization by HF patients in the local system. Thus, the adaptive practice of HF clinics retaining HF patients may have direct relevance to the integration of palliative care, because both cardiologists and family physicians in our study perceived that palliative care provision for HF patients was part of the family physician's role.

Our findings provide the first evidence of how HF care teams self-organize in nonlinear ways that nevertheless produce patterns at higher levels. As the last example suggests, some of these patterns have potential relevance for palliative care integration or, more broadly, care of those with chronic, complex medical illnesses. Based on our results, we contend that providing HF care consistent with palliative care principles requires both simple rules - as Zimmerman 
et al frame it, a "good-enough vision" - and the room for such simple rules to give rise to complex behaviors through emergence. ${ }^{21}$ As Sturmberg and Lanham argue, the goal is a health care delivery system that is locally adaptive and globally robust, allowing team members to interact and improvise in their local contexts. ${ }^{28}$ We contend that such simple rules should include understanding the advanced HF patient's unique needs and definition of quality of life, identifying and supporting a diverse HF team with many members in the community, recognizing when patients' unique needs render them unable to benefit from the usual system of care and recognizing that adaptations of that usual system arises from both patient characteristics and provider perspectives, tracking who is taking ownership for which aspects of a patient's care in any individual patient's team, and measuring success according to the expressed needs of a specific patient, rather than according to a one-size-fits-all model. This is not to diminish the importance of clinical practice guidelines, policies, and structures. These are crucial to provide the globally robust contribution; however, meeting unique patient needs in varied contexts inevitably requires improvisation and adaptation in how such are enacted in locally adaptive ways. As Zimmerman et al articulate of CAS, when it comes to structure or rules vs adaptivity and flexibility, that it is not either/or but both/and. ${ }^{21}$

Our study suggests that many of the most important needs and relationships of such complex medically ill patients exist within a social, community-based context, not within the walls of a hospital. In order to have simple rules give life to abundant emergent possibilities in this community-based context, team members need to be provided autonomy and support for their attempts to match a variety of resources to patient needs. Furthermore, team members, including health care providers and community team members, require explicit opportunities, through feedback loops, to influence practices within the local systems within which they work. Taking a relationship-based approach, informed through the lens of CAS, is one useful response to the wicked problem of tailoring globally robust best clinical practices to the reality of an individual patient and his or her reality in a locally adaptive context. Such an approach must inform not only health care delivery but also health professions education paradigms and the research methods we employ to improve our system.

With its nesting of teams and settings, health care for patients with HF presents an iconic instance of growing problem of coordinating care for the complex medically ill, otherwise referred to as those with multimorbidity and chronic disease. A dominant driver of health care quality and cost, such diseases interact with each other and cause needs to emerge in patients that are unique and far from one-sizefits-all. ${ }^{29,30}$ The tendency in health care has been to approach this problem with linear solutions, eg, standardization of practices such as referral and consultation, dissemination of evidence-based guidelines, and education of individuals regarding professional roles and responsibilities. ${ }^{31,32}$ However, our study of HF care teams illustrates that each of these solutions is challenged by the fluid, emergent, and nonlinear nature of daily team practice. Individuals do not provide care for the complex medically ill but CAS provide care. Such systems are not stable, predictable backdrops for linear interventions. While our results focus on the HF care context, the phenomenon of adaptive practices has relevance more broadly. It might usefully inform health care improvement scholarship in a range of clinical settings, such as geriatric care and mental health care, where we anticipate that similar adaptive practices underpin the variability of patient health care experience.

Our study employed a novel methodology well suited to examining the experiences of patients and their team members, the systems they are part of, and the interactions therein. Our sampling was primarily from heart function clinics, where the vast majority of NYHA class III and IV, or advanced, HF patients are followed. This may introduce a sampling bias, in that these patients may receive different care than those cared for by primary care physicians. We attempted to recruit from primary care settings as well, without sustained success. We purposively sampled patients who appeared to be underrepresented early in the data collection: we sought out more rural patients, patients with multiple comorbidities, and patients from a range of socioeconomic backgrounds. Our sampling strategy for creating TSUs was patient centered; patients defined their team members as the starting point for our examination of the system of HF team care. However, our reliance on semi-structured interviews with team members limits our ability to ascertain that accuracy of participant reports of their care experiences and we are unable to attribute intent to the practices our participants described. However, we contend that the description of adaptive practices should be judged based on its resonance with those practicing in the HF care domain and its ability to usefully query practices that tend to remain tacit and beyond critical reflection. Finally, our collection of data across five sites is both strength and a limitation. While it allowed insight into the diverse, locally contextualized experiences of patients and their team members, it is not designed for comparison, 
and we therefore do not propose to generalize our findings to other settings.

In summary, HF care teams, including patients and caregivers, exert regular effort to make things work in care provision. Such adaptive practices constitute a complicated backdrop for change efforts to integrate palliative care; it is difficult to intervene in a moving target. However, and more promisingly, they may also constitute a powerful new vehicle for change, if we can promote adaptive practices consistent with palliative care principles. Using the insights gained from CAS theory, we hope that local adaptations in keeping with palliative care may flourish in ways that linear, mechanistic interventions never could in the ever-shifting environment of advanced HF care.

\section{Acknowledgments}

The authors acknowledge the support from the Heart Failure/ Palliative Care Teamwork Research Group: Malcom Arnold, Fred Burge, Samuel Burnett, Karen Harkness, Gil Kimel, Donna Lowery, Denise Marshall, Robert McKelvie, Laura Nimmon, Joshua Shadd, Stuart Smith, and Donna Ward. The authors received financial support for the research of this article from the Canadian Institutes of Health Research and from AMOSO Innovations Fund.

\section{Disclosure}

The authors declared no potential conflicts of interest with respect to the research, authorship, and/or publication of this article.

\section{References}

1. Genest J, McPherson R, Frohlich J. 2009 Canadian Cardiovascular Society/Canadian guidelines for the diagnosis and treatment of dyslipidemia and prevention of cardiovascular disease in the adult. Can J Cardiol. 2009;25(10):567-579.

2. Hunt SA, Abraham WT, Chin MH, et al. ACC/AHA 2005 guideline update for the diagnosis and management of chronic heart failure in the adult. Circulation. 2005;112(12):154-235.

3. Jaarsma T, Beattie JM, Ryder M, et al; Advanced Heart Failure Study Group of the HFA of the ESC. Palliative care in heart failure: a position statement from the palliative care workshop of the Heart Failure Association of the European Society of Cardiology. Eur J Heart Fail. 2009;11(5):433-443.

4. McKelvie RS. The 2011 Canadian Cardiovascular Society heart failure management guidelines update: focus on sleep apnea, renal dysfunctional, mechanical circulatory support, and palliative care. Can J Cardiol. 2011;27:319-338.

5. WHO. Palliative Care; 2014. Available from: http://www.who.int/cancer/ palliative/en/.

6. Howlett J, Morin L, Fortin M, et al. End-of-life planning in heart failure: it should be the end of the beginning. Can J Cardiol. 2010;26(3): 135-141.

7. Hupcey JE, Penrod J, Fogg J. Heart failure and palliative care: implications in practice. $J$ Palliat Med. 2009;12(6):531-536.

8. Ryder M, Beattie JM, O'Hanlon R, McDonald K. Multidisciplinary heart failure management and end of life care. Curr Opin Support Palliat Care. 2011;5(4):317-321.
9. Fields AV, Kirkpatrick JN. Ethics of the heart: ethical and policy challenges in the treatment of advanced heart failure. Perspect Biol Med. 2012;55(1):71-80.

10. Light-McGroary L, Goodlin S. The challenges of understanding and managing pain in the heart failure patient. Curr Opin Support Palliat Care. 2012;7(1):14-20.

11. Lingard LA, McDougall A, Schulz V, et al. Understanding palliative care on the heart failure care team: an innovative research methodology. J Pain Symptom Manage. 2013;45(5):901.

12. Strachan PH, Kaasalainen S, Horton A, et al. Managing heart failure in the long-term care setting: nurses' experiences in Ontario, Canada. Nurs Res. 2014;63(5):357-365.

13. Jordan ME, Lanham HJ, Crabtree BF, et al. The role of conversation in health care interventions: enabling sensemaking and learning. Implement Sci. 2009;4(5):15.

14. Lanham HJ, McDaniel RR Jr, Crabtree BF, et al. How improving practice relationships among clinicians and nonclinicians can improve quality in primary care. Jt Comm J Qual Patient Saf. 2009;35(9):457-466.

15. Eoyang GH, Berkas TH. Evaluating Performance in a Complex, Adaptive System. Westport, CT: Quorum Books; 1999.

16. Marion R, Bacon J. Organizational extinction and complex systems. Emergence. 2000;1:71-96.

17. Plesk P. Redesiging Health Care with Insights from the Science of Complex Adaptive Systems. Washington, DC: National Academy Press; 2001.

18. Waldrop M. Complexity: The Emerging Science at the Edge of Order and Chaos. New York: Simon and Schuster; 1992.

19. Kauffman S. At Home in the Universe: The Search for Laws of SelfOrganization and Complexity. New York: Oxford University Press; 1995.

20. Stacey R. Ways of Thinking about Public Sector Governance. London: Routledge; 2006.

21. Zimmerman B, Lindberg C, Plesk P. Edgeware: Lessons from Complexity Science for Health Care Leaders. 0002 ed. United States of America: V H A, Incorporated; 2008.

22. Charmz K. Constructing Grounded Theory: A Practical Guide through Qualitative Analysis. 1st ed. London, UK: SAGE Publications; 2006.

23. Charmaz K. Constructing Grounded Theory (Introducing Qualitative Methods Series). 2nd ed. London, UK: SAGE Publications; 2014.

24. Dey I. Grounding Grounded Theory: Guidelines for Qualitative Inquiry. 1st ed. San Diego: Academic Press; 1999.

25. Munday DF, Johnson SA, Griffiths FE. Complexity theory and palliative care. Palliat Med. 2003;17:308-309.

26. Kannampallil TG, Schauer GF, Cohen T, Patel VL. Considering complexity in healthcare systems. J Biomed Inform. 2011;44(6):943-947.

27. Jones W. Complex Adaptive Systems; 2003. Available from: http://www. beyondintractability.org/essay/complex-adaptive-systems.

28. Sturmberg J, Lanham HJ. Understanding health care as a complex adaptive system. Achieving best possible health outcomes for individuals and communities by focusing on interdependencies. $J$ Eval Clin Pract. 2014;20(6):1005-1009.

29. Greene R, Dasso E, Ho S, Frank J, Scandrett G, Genaidy A. Patterns and expenditures of multi-morbidity in an insured working population in the United States: insights for a sustainable health care system and building healthier lives. Popul Health Manag. 2013;16(6):381-389.

30. Greene RA, Dasso E, Ho S, Genaidy A. A person focused model of care for the twenty-first century: a system-of-systems perspective. Popul Health Manag. 2014;17(3):166-171.

31. Chattoo S, Atkin K. Extending specialist palliative care to people with heart failure: semantic, historical and practical limitations to policy guidelines. Soc Sci Med. 2009;69(2):147-153.

32. Sturmberg J. Multimorbidity and chronic disease: an emergent perspective. J Eval Clin Pract. 2014;20:508-512. 


\section{Publish your work in this journal}

The Journal of Multidisciplinary Healthcare is an international, peerreviewed open-access journal that aims to represent and publish research in healthcare areas delivered by practitioners of different disciplines. This includes studies and reviews conducted by multidisciplinary teams as well as research which evaluates the results or conduct of such teams or

healthcare processes in general. The journal covers a wide range of areas and welcomes submissions from practitioners at all levels, from all over the world. The manuscript management system is completely online and includes a very quick and fair peer-review system. Visit http://www.dovepress.com/testimonials.php to read real quotes from published authors.

Submit your manuscript here: http://www.dovepress.com/journal-of-multidisciplinary-healthcare-journal 